\title{
Counterfactuals and accessibility
}

\author{
ABSTRACT. The accessibility relation between possible worlds can \\ be defined in the metalanguage of counterfactual semantics. As a \\ result, counterfactuals can ground the whole of standard modal \\ logic.
}

Necessity and possibility are often linked to counterfactuals via the following equivalences (Lewis 1973: 22, Stalnaker 1968: 105, Williamson 2007: 158):

(N) $\square A$ iff $\sim A \square \rightarrow A$

(P) $\diamond A$ iff $\sim(A \square \rightarrow \sim A)$

, where " $\square \rightarrow$ " denotes the counterfactual connective. ${ }^{1}$

$(\mathrm{N})$ and $(\mathrm{P})$ allow us to reduce modality to counterfactuals in the following sense: If $(\mathrm{N})$ and $(\mathrm{P})$ are axioms, then all modal truths can be derived from counterfactuals, and the language of boxes and diamonds can been seen as a fragment of the language of 'would' and 'might'. The general philosophical significance of this idea is that it can allay worries about the nature of, and our epistemic access to, modal truth. Assuming that counterfactual thinking grants reliable access to truths of a certain sort, reducing modal talk to counterfactual talk guarantees that there are genuine modal truths and that we have a reliable access to them. ${ }^{2}$

\footnotetext{
${ }^{1}$ (N) can be equivalently formulated as $\lceil\square A$ iff $\sim A \square \rightarrow \perp\rceil$.

${ }^{2}$ This is a bit crude, of course. See Williamson (2007: ch.5) for details.
} 
The goal of this paper is to show that the accessibility relation between possible worlds can also be recovered from counterfactuals, although by a slightly more indirect route. Specifically, I'll argue that the standard metalanguage of modal logic (the language of possible worlds related by a binary accessibility relation) is reducible to the metalanguage of (minimal change) counterfactual semantics. Very roughly, the claim is that we can take a world $u$ to be accessible from $w$ iff $u$ is one of the closest $A$-worlds, from the perspective of $w$, for some proposition $A$. The resulting relation of "counterfactual accessibility" will play the same semantic role with respect to modal axioms as the accessibility relation in standard modal logic.

This project is different from recovering modal truths via $(\mathrm{N})$ and $(\mathrm{P})$, because in the present case, the reduction takes place at the level of the respective metalanguages. I'll offer a few more thoughts on this contrast at the end of $\S 1$, after I sketch the main idea and its potential significance. Proofs are given in $\S 2$.

\section{Outline and motivation}

In standard modal semantics, properties of a binary accessibility relation $\alpha$ between worlds determine the validity of important modal axioms:

(1) $\lceil\square A \supset \diamond A\rceil$ is an axiom if $\alpha$ is serial (i.e. $\forall w \exists u$ : $\alpha(u, w)$ )

(2) $\lceil\square A \supset A\rceil$ is an axiom if $\alpha$ is reflexive

(3) $\lceil\square A \supset \square \square A\rceil$ is an axiom if $\alpha$ is transitive

(4) $\lceil A \supset \square \diamond A\rceil$ is an axiom if $\alpha$ is symmetric

All common modal systems can be assembled from these four axioms. For example, S4 is the system that contains (every substitution instance of) the first three axioms, while S5 is the system containing all four. 
(1)-(4) follow from the standard modal truth condition according to which $\square A$ is true at world $w$ iff $A$ is true at every world accessible from $w .^{3}$

My goal is to prove that $\alpha$ can be reduced to a relation that is definable in the metalanguage of counterfactual semantics. The proofs use (a generalized version) of the so-called minimal change theory of counterfactuals (cf. Nute and Cross 2002), the best-known version of which is the standard Lewis/Stalnaker semantics. Colloquially, $\lceil A \square \rightarrow B\rceil$ is true at $w$ in this setting iff $B$ is true at the $A$-worlds close to $w$. Slightly more formally, the core semantic idea is that $\lceil A \square \rightarrow B\rceil$ is evaluated at world $w$ by checking whether $B$ is true throughout an appropriate subset of $A$-worlds. What makes a subset appropriate is where different variants of minimal change semantics diverge. In $\S 2$, I'll give a generalized truth condition that fits all the main variants.

Bracketing the formal details for now, the proposal is to introduce a relation of counterfactual accessibility in the metalanguage of counterfactuals by taking world $u$ to be counterfactually accessible from $w$ iff $u$ is a member of the counterfactually relevant subset of $A$-worlds, for some proposition $A$. Colloquially, world $u$ is counterfactually accessible from world $w$ iff $u$ is one of the closest $A$-worlds (relative to $w$ ) for some proposition $A$. If all substitution instances of $(\mathrm{N})$ and $(\mathrm{P})$ are axioms, then the relation of counterfactual accessibility ("C $\alpha$ ") will have the following properties:

(C1) $\quad\lceil\square A \supset \diamond A\rceil$ is an axiom iff $\mathrm{C} \alpha$ is serial (i.e. $\forall w \exists u: \mathrm{C} \alpha(u, w)$ )

(C2) $\lceil\square A \supset A\rceil$ is an axiom iff $\mathrm{C} \alpha$ is reflexive

(C3) $\lceil\square A \supset \square \square A\rceil$ is an axiom iff $\mathrm{C} \alpha$ is transitive

(C4) $\lceil A \supset \square \diamond A\rceil$ is an axiom iff $\mathrm{C} \alpha$ is symmetric

3 For sophisticated proofs of (1)-(4), see Lemmon (1977: 50-55). Unsophisticated proofs are easy to construct by analogy with the right-to-left directions of Propositions $2-5$ in $\$ 2.1$. 
In other words, counterfactual accessibility plays the same role with respect to modal axioms as the accessibility relation in standard modal semantics. Consequently, no independent accessibility relation is needed once we have a minimal change model: the model supplies its own modal system. Proofs are given in $\S 2$. (Note that $(\mathrm{C} 1)-(\mathrm{C} 4)$ are biconditionals, and hence are stronger than (1)-(4). In principle, the latter can also be turned into biconditionals by analogy with Propositions $2-5$ in $\$ 2.1$.) I close this section with some thoughts on the potential significance of the proposal.

On the formal side, the present proposal makes it otiose to take a full Kripke frame to be a component of minimal change models, as e.g. Stalnaker (1968: 103) and Stalnaker and Thomason (1970: 25) do. Counterfactual accessibility dictates a modal structure in its own right, so assuming an independent Kripke frame either makes the resulting model contradictory (unless $(\mathrm{N})$ and $(\mathrm{P})$ are given up) or it is redundant. ${ }^{4}$

On the substantive side, the present project might offer a clue about the nature of accessibility. Accessibility relations are immensely useful for formal purposes, but it is hard to see, on the basis of standard modal semantics, what they are. Colloquially, they are sometimes defined by saying that $u$ is accessible from $w$ iff $w$ "sees" $u$, but this is just an aid to imagination, without any substantive content. If the present reduction is on the right track, then accessibility concerns interworld similarity. Since counterfactual closeness (which lies at the basis of counterfactual accessibility) is generally agreed to depend on overall similarities between worlds, the present reduction implies that the accessibility relation captures general features of the counterfactually relevant interworld similarity metric. Very roughly, world $u$ is accessible from $w$ iff is $u$ is overall very similar to $w$, under some counterfactual presupposition.

\footnotetext{
${ }^{4}$ Stalnaker and Thomason (1970: 27) stipulate that close worlds are always accessible (in terms of the independent Kripke frame). This approach avoids contradiction, but it is still redundant.
} 
The right-to-left directions of $(\mathrm{C} 1)-(\mathrm{C} 4)$ can help determine the correct modal logic for a given domain of discourse. If one has a domain of worlds (which do not yet qualify as possible or impossible at this initial stage), and, in addition, one has a similarity metric suitable for a minimal change semantics, then one already knows which (if any) of the standard modal systems the domain is a model of.

Conversely, the left-to-right directions of $(\mathrm{C} 1)-(\mathrm{C} 4)$ can help determine the counterfactually relevant similarity structure of a modal plenum that already has a modal system attached to it. E.g. if one has reason to believe that a given domain of modal discourse obeys S4 and not S5, and hence does not contain the axiom in (C4), then one knows that there are some worlds $u, w$ in the domain such that (speaking somewhat loosely) one can reach $w$ by reasoning counterfactually in the context of $u$ but not the other way around, because counterfactual accessibility is not symmetric.

The present reductive project is different from that of Williamson (2006: Ch.5, Appendix 1). He reduces modal truths to counterfactuals in the object language via $(\mathrm{N})$ and $(\mathrm{P})$, whereas in the present project, one reduces the metalanguage of modal logic to the metalanguage of counterfactual logic. This is hardly surprising: accessibility is a metalanguage notion. ${ }^{5}$ That said, one can scrap the machinery of minimal change semantics and carry out roughly the same type of reduction by using the single metalanguage notion of syntactic consequence. The price of this strategy is that one must allow infinitary conjunction and deduction in the object language (see §2.2).

\footnotetext{
${ }^{5}$ Williamson (2007: 300) derives $\lceil\square A \supset A\rceil$ in the object language from the axiom of weak centering, $\lceil(A \square \rightarrow B) \supset(A \supset B)\rceil$, and (on pp. 302-3) speculates about the status of the counterfactual equivalent of $\lceil\diamond A \supset \square \diamond A\rceil$. It is unclear (to me) how far this approach goes. Note that, in a minimal change setting, weak centering is standardly defined by the condition that for any $A$ that is true at $w, w$ is one of the $A$-worlds closest to $w$ (Lewis 1973: 120). This makes counterfactual accessibility reflexive, so $\lceil\square A \supset A\rceil$ follows by (C2).
} 


\section{Proofs}

\subsection{Minimal change semantics}

Notation

$\Omega=$ the set of worlds

$|A|=$ the set of worlds where proposition $A$ is true

$\Pi=$ the set of propositions

$\Pi(w)=$ the set of propositions true at world $w$

\section{Definitions}

$\square A={ }_{d f} \sim A \square \rightarrow A$

$A \diamond \rightarrow B$ ("If $A$ had been the case, $B$ might have been the case") $=_{d f}$

$=_{d f} \sim(A \square \rightarrow \sim B)$

$\diamond A=d f \quad A \diamond \rightarrow A$

\section{Assumptions}

(Biv) Worlds are bivalent: for any proposition $P,|P| \cap|\sim P|=\varnothing$ and $|P| \cup|\sim P|=\Omega$.

(Char) Worlds have characteristic propositions: For every world $w$, there is a proposition $P w$ such that $|P w|=\{w\}$.

N.B. This assumption is only needed to prove the left-to-right directions of Propositions 3 to 5 ( = (C2)-(C4) above).

(Sel) $\sigma(\bullet, \bullet)$ is a function from $\Pi \times \Omega$ to the powerset of $\Omega$ with the following properties:

$\left(\mathrm{Sel}_{1}\right) \quad$ For any proposition $P$ and world $w, \cup \sigma(P, w) \subseteq|P|$.

$\left(\mathrm{Sel}_{2}\right)$ For any propositions $P$ and $Q$ and world $w$, if $\cup \sigma(P, w)=\varnothing$, then $\cup \sigma(Q, w) \subseteq|\sim P|$. 


\section{Counterfactual truth}

$$
\begin{aligned}
& \lceil A \square \rightarrow B\rceil \text { is nonvacuously true at } w \text { iff } \exists S \in \sigma(A, w): \varnothing \neq S \subseteq|B| \\
& \lceil A \square \rightarrow B\rceil \text { is vacuously true at } w \text { iff } \cup \sigma(A, w)=\varnothing
\end{aligned}
$$

\section{Remark}

The following are theorems in all models that satisfy (Sel) and (T):

$$
\begin{aligned}
& A \square \rightarrow A \\
& \sim A \square \rightarrow A \quad \supset B \square \rightarrow A .
\end{aligned}
$$

Proof: (V1) is a direct consequence of (T) plus $\left(\mathrm{Sel}_{1}\right)$. (V2) is a direct consequence of (T) plus $\left(\mathrm{Sel}_{2}\right)$.

\section{Realizations of $\sigma(\bullet, \bullet)$}

(Sel) and (T) are meant to capture those general features of minimal change semantics that matter for present purposes. The precise nature of $\sigma(\bullet, \bullet)$ is different in each variant of minimal change semantics. In each case, one can give a definition of $\sigma(\bullet, \bullet)$ in terms of the relevant metalanguage notions so that $(\mathrm{T})$ turns out to be equivalent to the official truth condition of the theory in question. I sketch how this works in the case of the three main variants of minimal change semantics.

\section{Burgess (1981)}

The core metalanguage notion in Burgess's semantics is a three-term relation $R$ between worlds. Intuitively, $R w u t$ holds iff $u$ is at least as close to $w$ than $t$. In this framework, $\lceil A \square \rightarrow B\rceil$ is nonvacuously true at $w$ iff there is an $A$-world $t$ such that for any $u$, if $R w u t$, then $\lceil A \supset B\rceil$ is true at $u .\lceil A \square \rightarrow B\rceil$ is vacuously true at $w$ iff there is no $A$-world that is $R$-related to $w$. Here $\sigma(\bullet, \bullet)$ can be defined in two steps:

$$
\begin{aligned}
& S(w, t)=_{d f}\{u \in \Omega: R w u t\} \\
& \sigma(A, w)=_{d f}\{S(w, t) \cap|A|: t \in \Omega\}
\end{aligned}
$$


Using this definition, (T) will say that (i) $\lceil A \square \rightarrow B\rceil$ is nonvacuously true at $w$ iff there is an $A$-world $t$ such that $B$ is true at every $A$-world which is at least as close to $w$ as $t$, and (ii) $\lceil A \square \rightarrow B\rceil$ is vacuously true at $w$ iff no $A$-world is $R$-related to $w$. So the original truth original condition is recovered. It is easy to check that $\sigma(\bullet, \bullet)$, defined as above, satisfies (Sel).

Lewis (1973: 16)

Lewis works with "systems of spheres." For every world $w$, he posits a set $\$$, which contains (nested) sets of worlds, the "spheres of similarity" around $w$. These spheres indicate the relative distance of counterfactually relevant worlds from $w$. $\lceil A \square \rightarrow B\rceil$ is nonvacuously true at $w$ iff, for some $S \in \$_{w},\lceil A \supset B\rceil$ is true at all members of $S$, and at least one member of $S$ is an $A$-world. $\lceil A \square \rightarrow B\rceil$ is vacuously true at $w$ iff $\cup \$_{w} \subseteq|\sim A|$. Lewis's truth condition is equivalent to $(\mathrm{T})$ under the following definition:

$$
\sigma(A, w)=_{d f}\left\{S \cap|A|: S \in \$_{w}\right\}
$$

Stalnaker (1968)

In Stalnaker's system, a selection function $f(\bullet, \bullet)$ supplies, for each proposition $A$ and world $w$, the $A$-world closest to $w$. (Stalnaker assumes that there is always a single closest world. This assumption corresponds to the axiom of counterfactual excluded middle, cf. Lewis 1973: 79-81.) $\lceil A \square \rightarrow B\rceil$ is nonvacuously true at $w$ iff $B$ is true at $f(A, w)$, and vacuously true at $w$ iff $f(A, w)$ is "the absurd world" (an impossible world posited for this purpose). To recover these truth conditions via (T), one defines

$$
\begin{aligned}
\sigma(A, w)={ }_{d f}\{\{f(A, w)\}\} \text { if } f(A, w) \text { is not the absurd world, }\{\varnothing\} \\
\text { otherwise. }
\end{aligned}
$$




\section{Counterfactual accessibility}

(CAcc) $\quad \mathrm{C} \alpha(u, w) \equiv u$ is counterfactually-accessible from $w=_{d f}$ For some $A, u \in \cup \sigma(A, w)$.

NB. Counterfactual accessibility can also be defined in the following ways:

$\left(\mathrm{CAcc}_{1}\right) \quad \mathrm{C} \alpha(u, w)=_{d f} \cup \sigma(P u, w) \neq \varnothing$, where $P u$ is the characteristic proposition of $u$.

$\left(\mathrm{CAcc}_{2}\right) \quad \mathrm{C} \alpha(u, w)=_{d f}$ For some proposition $P,\lceil u$ obtains $\diamond \rightarrow P\rceil$ is true at $w$.

$\left(\mathrm{CAcc}_{3}\right) \quad \mathrm{C} \alpha(u, w)={ }_{d f}$ For some proposition $P,\left\lceil\left(\wedge_{Q \in \Pi(u)} Q\right) \diamond \rightarrow P\right\rceil$ is true at $w$.

$\left(\mathrm{CAcc}_{1}\right)$ is equivalent to (CAcc). Proof: By $\left(\mathrm{Sel}_{1}\right)$ and (Char), if $\cup \sigma(P u, w) \neq \varnothing$, then $\cup \sigma(P u, w)=\{u\}$, so if $u$ is accessible from $w$ in the sense of $\left(\mathrm{CAcc}_{1}\right)$, it is also accessible in the sense of (CAcc). In the other direction, if $u \in \cup \sigma(A, w)$ for some $A$, then $\cup \sigma(A, w) \not \subset|\sim P u|$, so by $\left(\operatorname{Sel}_{1}\right), \cup \sigma(P u, w) \neq \varnothing$.

The other two alternative definitions have additional presuppositions. $\left(\mathrm{CAcc}_{2}\right)$ presupposes that worlds have names in the object language, while $\left(\mathrm{CAcc}_{3}\right)$ presupposes that the object language tolerates infinitary conjunction.

Given the relevant presupposition, the equivalence of $(\mathrm{CAcc})$ and $\left(\mathrm{CAcc}_{2}\right)$ is easy to establish by setting $P=\lceil u$ obtains $\rceil$ and noting that, by $(\mathrm{T}),($ Sel), and Lemma 1 (see below), $u \in \cup \sigma(P, w)$ iff $\lceil P \diamond \rightarrow P\rceil$ is true at $w$. The equivalence of (CAcc) and $\left(\mathrm{CAcc}_{3}\right)$ can be shown the same way by setting $P=\left(\wedge_{Q \in \Pi(u)} Q\right)$.

\section{Lemma 1}

$\lceil\square A\rceil$ is true at $w$ iff $\cup \sigma(\sim A, w)=\varnothing$.

Left-to-right: $\lceil\square A\rceil$ is equivalent to $\lceil\sim A \square \rightarrow A\rceil$, which, by (Biv) and (T), can only be vacuously true at any world. So $\cup \sigma(\sim A, w)=\varnothing$ whenever $\lceil\square A\rceil$ is true at $w$. Right-to-left: Direct consequence of (T) and the definition of $\lceil\square A\rceil$. 


\section{Lemma 2}

$\lceil\square A\rceil$ is true at $w$ iff for any proposition $P, \cup \sigma(P, w) \subseteq|A|$.

Left-to-right: By Lemma 1, if $\lceil\square A\rceil$ is true at $w$, then $\cup \sigma(\sim A, w)=\varnothing$, which, by $\left(\operatorname{Sel}_{2}\right)$, entails that $\cup \sigma(P, w) \subseteq|A|$ for any $P$.

Right-to-left: If the right-hand side is true, then $\cup \sigma(\sim A, w) \subseteq|A|$. By (Biv) and $\left(\operatorname{Sel}_{1}\right), \cup \sigma(\sim A, w)=\varnothing$, so by Lemma $1,\lceil\square A\rceil$ is true at $w$.

\section{Proposition 1}

$\lceil\square A\rceil$ is true at $w$ iff For every $u$ that is counterfactually-accessible from $w, u \in|A|$.

Left-to-right: Let $u$ be counterfactually-accessible from $w$. Then for some $P$, $u \in \cup \sigma(P, w)$. By Lemma 2 and given the left-hand side, $\cup \sigma(P, w) \subseteq|A|$ and so $u \in|A|$.

Right-to-left: It follows from the right-hand side that for any proposition $P$, $\cup \sigma(P, w) \subseteq|A|$. Suppose that $P=\sim A$ and consider $\sigma(\sim A, w)$. If $\cup \sigma(\sim A, w) \neq \varnothing$, i.e. if $\lceil\sim A \square \rightarrow \sim A\rceil$ is nonvacuously true at $w$, then by $\left(\operatorname{Sel}_{1}\right)$ and the right-hand side, $\varnothing \neq \cup \sigma(\sim A, w) \subseteq|A| \cap|\sim A|$, which contradicts (Biv). So $\cup \sigma(\sim A, w)=\varnothing$ and hence by Lemma $1,\lceil\square A\rceil$ is true at $w$.

\section{Corollary 1}

(a) $\lceil\diamond A\rceil$ is true at $w$ iff there is a world $u$ such that $\mathrm{C} \alpha(u, w)$ and $u \in|A|$.

(b) $\lceil\square \square A\rceil$ is true at $w$ iff for every world $u$ and world $t$ such that $\mathrm{C} \alpha(u, w)$ and $\mathrm{C} \alpha(t, u), t \in|A|$.

(c) $\lceil\square \diamond A\rceil$ is true at $w$ iff for every world $u$ such that $\mathrm{C} \alpha(u, w)$, there is a world $t$ such that $\mathrm{C} \alpha(t, u)$ and $t \in|A|$. 


\section{Proposition 2}

$\lceil\square A \supset \diamond A\rceil$ is true for all $A$ at all $w$ iff counterfactual accessibility is serial (i.e. iff $\forall w \exists u$ : $\mathrm{C} \alpha(u, w)$ ).

Right-to-left: Suppose that $\lceil\square A\rceil$ is true at $w$. Given that $\mathrm{C} \alpha$ is serial, $\mathrm{C} \alpha(u, w)$ for some $u$. By Proposition $1, u \in|A|$, so by Corollary $1 \mathrm{a},\lceil\diamond A\rceil$ is true at $w$. Left-to-right: Take an arbitrary proposition $A$. If $\lceil\square A\rceil$ is false at $w$, then, by Proposition 1 , some $\sim A$-world is counterfactually accessible from $w$. If $\lceil\square A\rceil$ is true at $w$, then, by the left-hand side, so is $\lceil\diamond A\rceil$, and hence, by Proposition 1 , some $A$-world is counterfactually accessible from $w$.

\section{Proposition 3}

$\lceil\square A \supset A\rceil$ is true for all $A$ at all $w$ iff counterfactual accessibility is reflexive.

Right-to-left: Suppose that $\lceil\square A\rceil$ is true at $w$. By Proposition 1, it follows that $u \in|A|$ whenever $\operatorname{C} \alpha(u, w)$. Since $\mathrm{C} \alpha(w, w)$, it follows that $\lceil A\rceil$ is true at $w$. Left-to-right: Let $P w$ be the characteristic proposition of $w$. Because of the left-hand side, $\lceil P w \supset \diamond P w\rceil$ is true at $w$, and hence $\lceil\diamond P w\rceil$ is true at $w$, hence by Proposition 1, some world where $P w$ is true is counterfactually-accessible from $w$. But the only such world is $w$. So $\mathrm{C} \alpha(w, w)$.

\section{Proposition 4}

$\lceil\square A \supset \square \square A\rceil$ is true for all $A$ at all $w$ iff counterfactual accessibility is transitive.

Right-to-left: Suppose that $\lceil\square A\rceil$ and $\lceil\sim \square \square A\rceil$ are true at $w$. By Corollary $1 \mathrm{~b}$, the latter entails that for some world $u$ and world $t$ such that $\mathrm{C} \alpha(u, w)$ and $\mathrm{C} \alpha(t, u), t \in|\sim A|$. By transitivity, $\mathrm{C} \alpha(t, w)$. By Proposition 1 and given that 
$\lceil\square A\rceil$ is true at $w$, it follows that $t \in|A|$, and hence $|\sim A| \cap|A| \neq \varnothing$, which contradicts (Biv). Therefore $\lceil\square A \supset \square \square A\rceil$ is true at $w$. Left-to-right: Suppose that $\mathrm{C} \alpha(u, w), \mathrm{C} \alpha(t, u)$, and $\sim \mathrm{C} \alpha(t, w)$. Because of the last condition, $\lceil\square \sim P t\rceil$ is true at $w$, where $P t$ is the characteristic proposition of $t$. (Proof: If $\lceil\diamond P t\rceil$ were true at $w$, it would follow by Proposition 1 that a $P t$-world is accessible from $w$, but the only such world is $t$.) By the left-hand side, it follows that $\lceil\square \square \sim P t\rceil$ is true at $w$, hence by Corollary $1 \mathrm{~b},\lceil\square \sim P t\rceil$ is true at $u$, contradicting the assumption that $\mathrm{C} \alpha(t, u)$. So $\mathrm{C} \alpha(t, w)$.

\section{Proposition 5}

$\lceil A \supset \square \diamond A\rceil$ is true for all $A$ at all $w$ iff counterfactual accessibility is symmetric.

Right-to-left: By Corollary 1c, $\lceil\square \diamond A\rceil$ is true at $w$ iff for every world $u$ such that $\mathrm{C} \alpha(u, w)$, there is a world $t$ such that $\mathrm{C} \alpha(t, u)$ and $t \in|A|$. Suppose that $w \in|A|$ and $\mathrm{C} \alpha(u, w)$. Given that counterfactual accessibility is symmetric, $\mathrm{C} \alpha(w, u)$, and so $w$ itself can serve as the $t$ in the truth condition of $\lceil\square \diamond A\rceil$. Therefore $\lceil A \supset \square \diamond A\rceil$ is true at $w$. Left-to-right: Suppose that $\mathrm{C} \alpha(u, w)$, and take $P w$, the characteristic proposition of $w$. Because of the left-hand side, $\lceil\square \diamond P w\rceil$ is true at $w$, so by Corollary $1 \mathrm{c},\lceil\diamond P w\rceil$ is true at $u$, so by Proposition 1 , there is a $P w$-world accessible from $u$. But the only such world is $w$. So $\mathrm{C} \alpha(w, u)$. 


\subsection{Propositional logic with 'worlds'}

The goal of this section is to show that the main results of $\$ 2.1$ (viz. Propositions 1 to 5) are to some extent independent of minimal change semantics: they can be proved in a very austere semantic framework where syntactic consequence is the only relevant metalanguage notion. Maximal consistent propositions go proxy for worlds in this setting. The framework itself is semantically not too edifying, because it treats counterfactual truth as primitive, but it helps one see that the reduction is driven by the two counterfactual axioms that (Sel) and (T) validate (see (V1) and (V2) below and cf. the Remark after (T) in §2.1). The price one pays for the simplicity of the metalanguage is that infinitary conjunction and deduction must be allowed in the object language.

\section{Assumptions}

(MaxCon) 'Worlds' are maximal consistent propositions: for any 'world' $W$ and any proposition $P, W \mid-P$ xor $W \mid-\sim P$.

(Plenitude) Every non-self-contradictory proposition 'obtains' at a specific set of 'worlds': for any proposition $P, P \mid \sim P$ xor there are some 'worlds' $W_{\mathrm{i}}$ such that $P \mid-\mathrm{v}_{\mathrm{i}} W_{\mathrm{i}}$ (note that, by (MaxCon), $W_{\mathrm{i}}-P$ for all $i$ in the latter case).

\section{Definitions}

$\square A=_{d f} \sim A \square \rightarrow A$

$$
A \diamond \rightarrow B=_{d f} \sim(A \square \rightarrow \sim B)
$$

\section{Counterfactual accessibility*}

$\mathrm{C} \alpha^{*}(U, W) \equiv$ 'world' $U$ is counterfactually-accessible* from 'world' $W=_{d f}$ $W+(U \diamond \rightarrow U)$ 
Rules

(Ded) Deduction within counterfactuals:

If there are (arbitrarily many) propositions $B_{\mathrm{i}}$ such that $\vdash A \square \rightarrow B_{i}$ (for all $i$ ) and $\mid-\left(\wedge_{\mathrm{i}} B_{\mathrm{i}}\right) \supset C$, then $\mid-A \square \rightarrow C$.

Modus ponens

Interchange of logical equivalents

\section{Axioms}

(V1) $\quad A \square \rightarrow A$

(V2) $\sim A \square \rightarrow A \supset B \square \rightarrow A$.

\section{Lemma 3}

If $W \mid-\sim(A \square \rightarrow C)$ and $B \mid-C$, then $W \mid-\sim(A \square \rightarrow B)$.

Proof: Suppose that the antecedent is true. By (MaxCon), either $W \mid \sim(A \square \rightarrow B)$ or $W \mid-(A \square \rightarrow B)$. Suppose the latter is true. Then by (Ded) and given that $B|C, W|-(A \square \rightarrow C)$, contradicting (MaxCon). So $W \mid \sim(A \square \rightarrow B)$.

\section{Proposition 6}

$W \vdash \square A$ iff for every $U$ counterfactually-accessible* from $W, U \vdash A$.

Left-to-right: Suppose that the left-hand side is true. If the right-hand side is false, then for some $U, W \mid(U \diamond \rightarrow U)$ and (by MaxCon) $U-\sim A$.

By (V1), $W \mid-(U \square \rightarrow U)$, and by (Ded) and given that $U \mid-\sim A$, $W \mid(U \square \rightarrow \sim A)$. By (V2) and the left-hand side, $W \mid-(U \square \rightarrow A)$. By (Ded) and given that $(A \wedge \sim A)|-\sim U, W|-(U \square \rightarrow \sim U)$, contradicting the hypothesis that $U$ is counterfactually-accessible* from $W$. 
Right-to-left: Suppose that the right-hand side is true. By (Plenitude), either $\sim A \mid-A$ or $\sim A \mid-\vee_{\mathrm{i}} U_{\mathrm{i}}$ for some 'worlds' $U_{\mathrm{i}}$ such that $U_{\mathrm{i}} \mid-\sim A$ for all $i$. If the former is the case, then by (V1), $W \mid-(\sim A \square \rightarrow \sim A)$ and by (Ded), $W \mid$ $(\sim A \square \rightarrow A)$, i.e. $W \mid \square A$. On the second horn, we know from the righthand side and (MaxCon) that $W-\left(U_{\mathrm{i}} \square \rightarrow \sim U_{\mathrm{i}}\right)$ for all $i$. By (V2), $W \mid$ $\left(\sim A \square \rightarrow \sim U_{\mathrm{i}}\right)$ for all $i$, and by (Ded), $W \mid-\left(\sim A \square \rightarrow \wedge_{\mathrm{i}} \sim U_{\mathrm{i}}\right)$. By (MaxCon), either $W \mid-\left(\sim A \square \rightarrow \vee_{\mathrm{i}} U_{\mathrm{i}}\right)$ or $W \mid \sim\left(\sim A \square \rightarrow \vee_{\mathrm{i}} U_{\mathrm{i}}\right)$. If the former, then, by (Ded) and the fact that $\left[\left(\wedge_{\mathrm{i}} \sim U_{\mathrm{i}}\right) \wedge\left(\vee_{\mathrm{i}} U_{\mathrm{i}}\right)\right] \mid-A$, it follows that $W \mid-\square A$. On the second horn, by Lemma 3 and the fact that $\sim A \mid \vee_{\mathrm{i}} U_{\mathrm{i}}$, it follows that $W \mid \sim(\sim A \square \rightarrow \sim A)$, contradicting (V1). So $W \mid \square A$.

\section{Remark 2}

Using Proposition 6, analogues of Corollary 1 and Propositions 2 to 5 are straightforward to prove.

\section{References}

Burgess, John P. (1981): Quick completeness proofs for some logics of conditionals. Notre Dame Journal of Formal Logic 22: 1, 76-84.

Lemmon, E. J. (1977): An Introduction to Modal Logic. American Philosophical Quarterly Monographs.

Lewis, David (1973): Counterfactuals. Blackwell.

Nute, Donald and Charles B. Cross (2002): Conditional logic. In D. M. Gabbay and F. Guenthner (eds), Handbook of Philosophical Logic (2nd ed.) IV, 1-98.

Stalnaker, Robert C. (1968): A theory of conditionals. American Philosophical Quarterly Monographs 2, 98-112.

Stalnaker, Robert C. and Richmond H. Thomason (1970): A semantic analysis of conditional logic. Theoria 36: 1, 23-42.

Williamson, Timothy (2007): The Philosophy of Philosophy. Blackwell. 